\title{
IR OBSERVATIONS OF THE K AND F CORONA DURING THE 1991 ECLIPSE
}

\author{
J. R. KUHN and H. LIN \\ Michigan State University, East Lansing, MI 48824, U.S.A. \\ P. LAMY \\ Laboratoire d'Astronomie Spatiale, F-13012 Marseille, France \\ S. KOUTCHMY \\ Institut d'Astrophysique, Boulevard Arago, F-75014 Paris, France \\ and \\ R. N. SMARTT \\ National Solar Observatory, Sunspot, NM 88349, U.S.A.
}

\begin{abstract}
The availability of relatively large format IR array detectors is incentive for reexamining the classic question of whether or not there are "dust rings" around the sun - a problem for which there are conflicting observational answers. The 1991 eclipse path included a high altitude observatory and provided a potentially ideal opportunity to study the infrared properties and dust content of the corona. Here we report results from an experiment conducted from Mauna Kea using a $\mathrm{HgCdTe}$ array detector sensitive to wavelengths between 1-2.5 $\mu \mathrm{m}$. Surface brightness measurements in the H-band and polarization data in the J-band were obtained over a field-ofview of $\pm 6 R_{\odot}$ while $\mathrm{K}$-band images further extend to $15 R_{\odot}$ on the western side of the Sun. $\mathrm{J}$-band polarization data and $\mathrm{H}$ and $\mathrm{K}$-band surface brightness data clearly show the inhomogeneous structure in the $\mathrm{K}$ corona and the ecliptical flattening of the $\mathrm{F}$ corona. We see no evidence of a circumsolar, local dust corona (dust rings) out to $15 R_{\odot}$.
\end{abstract}

Key words: eclipses - infrared: stars - interplanetary medium - Sun: corona

\section{Introduction}

The Fraunhofer or F corona is that component of the surface brightness with absorbtion lines in its spectrum. Unlike the $\mathrm{K}$ corona, which has no spectral features and results primarily from photospheric light scattered by hot coronal electrons, the F component results from sunlight scattered off of zodiacal dust. It is unclear to what extent this component results from light scattered by dust in the immediate environment of the sun's corona. The F-corona may simply be "zodiacal" light, or photons scattered by grains far from the sun but close to the line-of-sight to the corona.

Peterson (1963) was one of the first to suggest that it should be possible to look for a "local" coronal contribution to the $\mathrm{F}$ component by looking for the thermal emission of hot dust in the outer corona. In this model dust may be deposited in the inner solar system by comets. Small grains that orbit the sun should then experience a drag force as they scatter or reradiate solar radiation (cf. Wyatt and Whipple 1950). Thus a grain gradually spirals toward the sun until it reaches its sublimation temperature. At this point the solar radiation force to gravitational force ratio increases and the grain either vaporizes or evolves into an elliptic orbit that takes it away from the sun. The inner circumsolar radius within which there are no grains depends on the grain properties. Silicate grains with radii of a few microns should survive for a few hundred orbits near 4 solar radii (cf. Mukai 1985) 
and grains that start out much smaller will not get close to the sun. Several authors have reported observations of the thermal and large-angle scattered light from such dust rings, starting with Peterson (1963) and MacQueen (1968). None of these or later reports have been entirely consistent ( $c f$. Koutchmy and Lamy 1985) and others have been unable to detect any local F-coronal light contribution (Mampaso et al. 1982). While these are difficult observations it seems clear that a definitive observation of the local $\mathrm{F}$ component will significantly constrain our understanding of the solar environment.

Since the sublimation temperature of the grains is expected to be near $2000 \mathrm{~K}$, the thermal emission should peak at a wavelength between 1 and $2 \mu \mathrm{m}$ and the thermal emission throughout the circumsolar dust region should be redder than the Thomson-scattered $\mathrm{K}$ coronal flux component. In addition a local $\mathrm{F}$ corona component resulting from large-angle scattering from dust grains should show some linear polarization. Clearly an experiment designed to detect this light should incorporate polarization and infrared surface brightness observations. Until the 1991 eclipse, all prior IR experiments used scanning single-element detectors to look for excess surface brightness "bumps" along the ecliptic. During a typical eclipse experiment, where there are only a few seconds or minutes of totality, a large angular region to be mapped, and under conditions of a changing background sky brightness - these are difficult observations. It is evident that a sensitive array detector experiment may make the identification of dust-related surface brightness enhancements considerably easier since each pixel records the coronal surface brightness simultaneously.

\section{The Experiment}

The detector is a $128 \times 128 \mathrm{HgCdTe}$ array device, produced by Rockwell International (part TCM-1000C). Each pixel has a large photoelectron well capacity $\left(3 \times 10^{7}\right)$ with a read noise of about 2000 electrons. Our detector has an average quantum efficiency of about $70 \%$ for light with wavelengths between 1 and $2.5 \mu \mathrm{m}$. The array operates at liquid nitrogen temperature, behind cold filters, polarizers, and objective lens. The device is electronically shuttered so it does not require a fast mechanical shutter to define the exposure time. Figure 1 shows a schematic of the eclipse experiment. Standard (Barr Associates Inc.) infrared $\mathrm{H}$ and $\mathrm{K}$ filters are mounted on a cold filter wheel. Three J band filters with type HR (Polaroid, Inc.) sheet polarizers are also mounted on the cooled wheel assembly. The filter wheel is controlled by the experiment computer and a motor outside the dewar. The $110 \mathrm{~mm}$ f.l., f/ 2 (stopped down to $\mathrm{f} / 4$ during the experiment) doublet objective was mounted in the dewar. Because of the large wavelength range of the observations we used an internally mounted stepper motor to make focus adjustments. The final image scale ranged between 0.094 and $0.098 R_{\odot}$ per pixel. The detector system is described more fully elsewhere (McPherson et al. 1992).

The field-of-view of the detector is about 3.2 degrees or $\pm 6 R_{\odot}$ so that the corona will be about $10^{3}$ times brighter near the limb of the moon than it is at the edge of our field. Laboratory measurements show that there is scattered light in the optics, probably due to multiple reflections from the lens, at a level near 


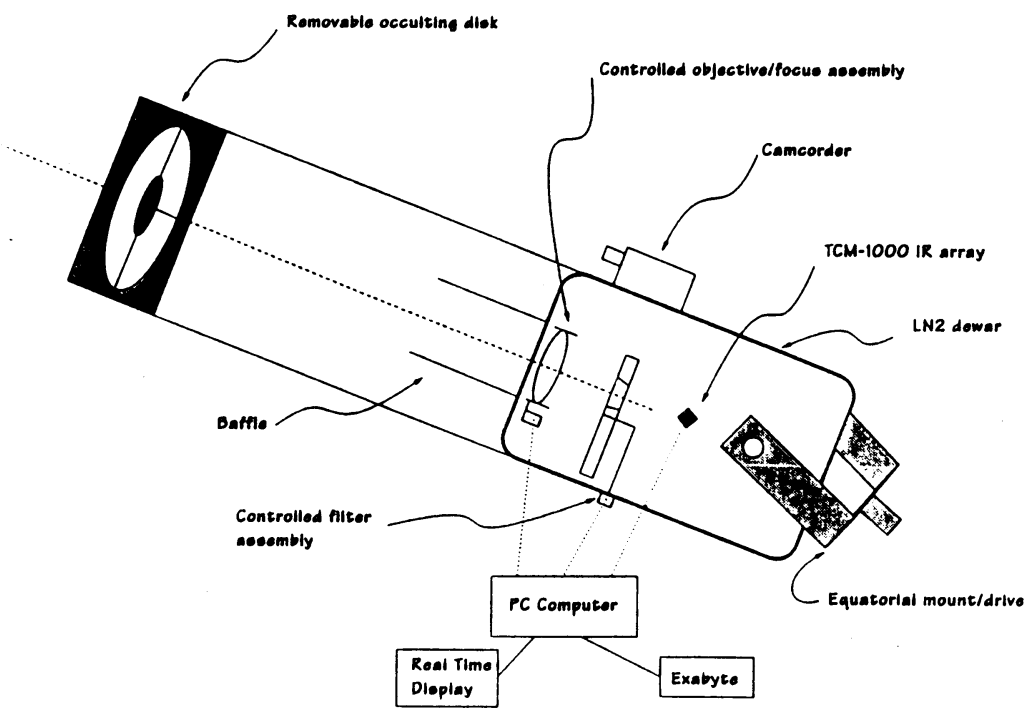

Fig. 1. Schematic of the principle components of the eclipse experiment.

$10^{-3}$ of the surface brightness at the center of the field. Unfortunately this residual signal changes with the illumination pattern, filter, and focus, and so it cannot easily be modeled. Our solution to this problem was to externally occult the bright inner corona. We accomplish this with a mask approximately $1.2 \mathrm{~m}$ in front of the focal plane. The mask supports a removable 3 -cm radius occulting disk that can be positioned in a $6-\mathrm{cm}$ radius opening. With the central disk removed the telescope may also be tilted away from the sun to see the outer corona - so that the bright inner corona does not illuminate the telescope optics. The thermal emission of the occulting disk at the K-band was less than the sky background signal and is not a problem.

The dewar and occulter assembly were mounted directly to a simple equatorial sun-tracker. The equatorial mount was driven at a fixed rate, but allowed for rapid manual offsets in RA and Dec. During the eclipse we manually shifted the telescope to the west to see the outer corona. Since the moon's limb was not in the field of the IR array during the offset observations we mounted a camcorder with a large field of view on the tracker. Thus we have a video record that could be digitized to measure the precise spatial offset of the image when the limb was not visible on the array.

The filter/polarizer wheel, focus mechanism, and array were controlled by a PC. Images could be read, flat-fielded and displayed in real time, while reading and recording them to Exabyte tape required about $1 \mathrm{~s}$. We had an ambitious observing run planned for the 4 minutes of totality which required a computer script to run smoothly. During this time we would observe $\mathbf{J}$ polarization and $\mathbf{H}$ and $\mathrm{K}$ flux both with, and without the occulter. In addition we would offset the 
telescope to observe the $\mathrm{K}$ flux beyond $14 R_{\odot}$. In order to observe the inner corona without saturating the detector we also needed exposures as short as $3.8 \mathrm{~ms}$, and for measuring the outer corona our longest exposure was $10 \mathrm{~s}$. The largest "overhead" in the observations was the time spent manually shifting the telescope and the time required by the internal stepper-motor to change the focus for the K-band data (about $15 \mathrm{~s}$ ). Table I shows a log of the eclipse data - the sequence number, filter bandwidth, exposure duration, time, and occulter position.

\section{Analysis}

The IR array images were calibrated like most optical CCD data. Flat-fields were obtained separately for each of the polarization angles, occulter positions and wavelengths from blank sky observations. Because the Mauna Kea sky clouded over soon after the eclipse we used flat-field data that were obtained the previous day. Amplifier bias and dark field corrections were obtained from "overscan" data and dewar "cold stop" observations. The field-flattened flux data were calibrated using observations of Vega and Altair obtained on the night of July 9. Unfortunately the eclipse sky conditions were far from photometric and we estimate that our overall flux calibration may be in error by as much as $60 \%$.

An image of the "sky" can be produced by looking at the difference between two eclipse images obtained in rapid succession near second or third contact - when the overall illumination level is changing rapidly. Since the unscattered coronal light does not vary (except at the limb of the moon!) on a timescale of a second or less, a difference image will show primarily the change in the scattered light caused by both clouds and our optics. The difference of externally occulted images shows the change in scattered light caused by clouds. Thus the changing lunar limb position gives an independent check on the scattered light in our measurements. Figure 2 shows a difference image taken using H-band data obtained at 43 and 46 seconds after 2nd contact. A similar wavy pattern, near the edges of the frame, also appears in occulted images and so is apparently due to atmospheric scattered light. It has an rms amplitude of about $1.7 \times 10^{-3}$ of the change in the $\mathrm{H}$ brightness at the lunar limb. Figure 2 shows the relative scattered light in the $\mathrm{H}$ and $\mathrm{K}$ band data, normalized by the brightness change observed near the lunar limb. Within $1.7 R_{\odot}$ the long exposure data is saturated - requiring that they be normalized (indirectly) by the product of the measured time rate of change in the integrated coronal brightness, the peak limb brightness, and the time between exposures. Thus, Figure 3 is a direct measure of the scattered-light solar aureole in the $\mathrm{H}$ and $\mathrm{K}$ bands, normalized by the average surface brightness near the lunar limb.

The calibrated, circularly averaged, $\mathrm{H}$ and $\mathrm{K}$ surface brightness is plotted in Figure 4. In these units solar color implies a $\mathrm{K}$ band flux which is a factor of about 3 less than the $\mathrm{H}$ flux (which is what we observe near the limb). Between 1 and $7 R_{\odot}$ the $\mathrm{H}$ and $\mathrm{K}$ brightness decline by a factor of about 300 and 500 respectively. Using the measured limb brightness and scattering function profile from Figure 3 we can estimate the scattered light component in the data, which is also plotted on Figure 4. Figure 3 shows that the scattered light declines more rapidly in the longer wavelength band, i.e., that the scattered light gets bluer further 
TABLE I

IR Observing Log for July 11, 1991 Eclipse

\begin{tabular}{|c|c|c|c|c|}
\hline Seq. No. & Filter & Occulter & Exp. Time & Start Time \\
\hline 01 & $\mathrm{~K}$ & NO & 0.0038 & $17: 28: 19$ \\
\hline 02 & $\mathrm{~K}$ & NO & 0.5 & $17: 28: 21$ \\
\hline 03 & $\mathrm{H}$ & NO & 0.0038 & $17: 28: 48$ \\
\hline 04 & $\mathrm{H}$ & NO & 0.0038 & $17: 28: 50$ \\
\hline 05 & $\mathrm{H}$ & NO & 0.5 & $17: 28: 52$ \\
\hline 06 & $\mathrm{H}$ & NO & 0.5 & $17: 28: 55$ \\
\hline 07 & $\mathrm{~J} 1$ & NO & 0.0038 & $17: 29: 05$ \\
\hline 08 & $\mathrm{~J} 1$ & NO & 0.0038 & $17: 29: 07$ \\
\hline 09 & $\mathrm{~J} 1$ & NO & 0.5 & $17: 29: 09$ \\
\hline 10 & $\mathrm{~J} 1$ & NO & 0.5 & $17: 29: 11$ \\
\hline 11 & $\mathrm{~J} 2$ & NO & 0.0038 & $17: 29: 18$ \\
\hline 12 & $\mathrm{~J} 2$ & NO & 0.0038 & $17: 29: 20$ \\
\hline 13 & $\mathrm{~J} 2$ & NO & 0.5 & $17: 29: 22$ \\
\hline 14 & $\mathrm{~J} 2$ & NO & 0.5 & $17: 29: 24$ \\
\hline 15 & $\mathrm{~J} 3$ & NO & 0.0038 & $17: 29: 31$ \\
\hline 16 & $\mathrm{~J} 3$ & NO & 0.0038 & $17: 29: 32$ \\
\hline 17 & $\mathrm{~J} 3$ & NO & 0.5 & $17: 29: 34$ \\
\hline 18 & $\mathrm{~J} 3$ & NO & 0.5 & $17: 29: 37$ \\
\hline 19 & $\mathrm{~J} 3$ & YES & 2.5 & $17: 29: 44$ \\
\hline 20 & $\mathrm{~J} 3$ & YES & 2.5 & $17: 29: 48$ \\
\hline 21 & $\mathrm{~J} 3$ & YES & 2.5 & $17: 29: 53$ \\
\hline 22 & $\mathrm{~J} 2$ & YES & 2.5 & $17: 30: 01$ \\
\hline 23 & $\mathrm{~J} 2$ & YES & 2.5 & $17: 30: 05$ \\
\hline 24 & $\mathrm{~J} 2$ & YES & 2.5 & $17: 30: 10$ \\
\hline 25 & $\mathrm{~J} 1$ & YES & 2.5 & $17: 30: 18$ \\
\hline 26 & $\mathrm{~J} 1$ & YES & 2.5 & $17: 30: 22$ \\
\hline 27 & $\mathrm{~J} 1$ & YES & 2.5 & $17: 30: 27$ \\
\hline 28 & $\mathrm{H}$ & YES & 2.5 & $17: 30: 39$ \\
\hline 29 & $\mathrm{H}$ & YES & 2.5 & $17: 30: 43$ \\
\hline 30 & $\mathrm{H}$ & YES & 2.5 & $17: 30: 48$ \\
\hline 31 & $\mathrm{~K}$ & YES & 2.5 & $17: 31: 17$ \\
\hline 32 & $\mathrm{~K}$ & YES & 2.5 & $17: 31: 22$ \\
\hline 33 & $\mathrm{~K}$ & YES & 2.5 & $17: 31: 25$ \\
\hline 34 & $\mathrm{~K}$ & NO & 0.0038 & $17: 31: 32$ \\
\hline 35 & $\mathrm{~K}$ & NO & 0.0038 & $17: 31: 34$ \\
\hline 36 & $\mathrm{~K}$ & NO & 0.5 & $17: 31: 36$ \\
\hline 37 & $\mathrm{~K}$ & NO & 0.5 & $17: 31: 39$ \\
\hline 38 & $\mathrm{~K}$ & OFFSET & 1.5 & $17: 31: 53$ \\
\hline 39 & $\mathrm{~K}$ & OFFSET & 1.5 & $17: 31: 56$ \\
\hline 40 & $\mathrm{~K}$ & OFFSET & 1.5 & $17: 32: 00$ \\
\hline 41 & $\mathrm{~K}$ & OFFSET & 10.5 & $17: 32: 03$ \\
\hline
\end{tabular}

Column 1 identifies the sequence number; column 2 shows the filter bandwidth; column 3 indicates whether the occulter was in place or if the telescope was offset to the west; column 4 is the exposure time in seconds; column 5 shows the start time (UT) of the exposure. 


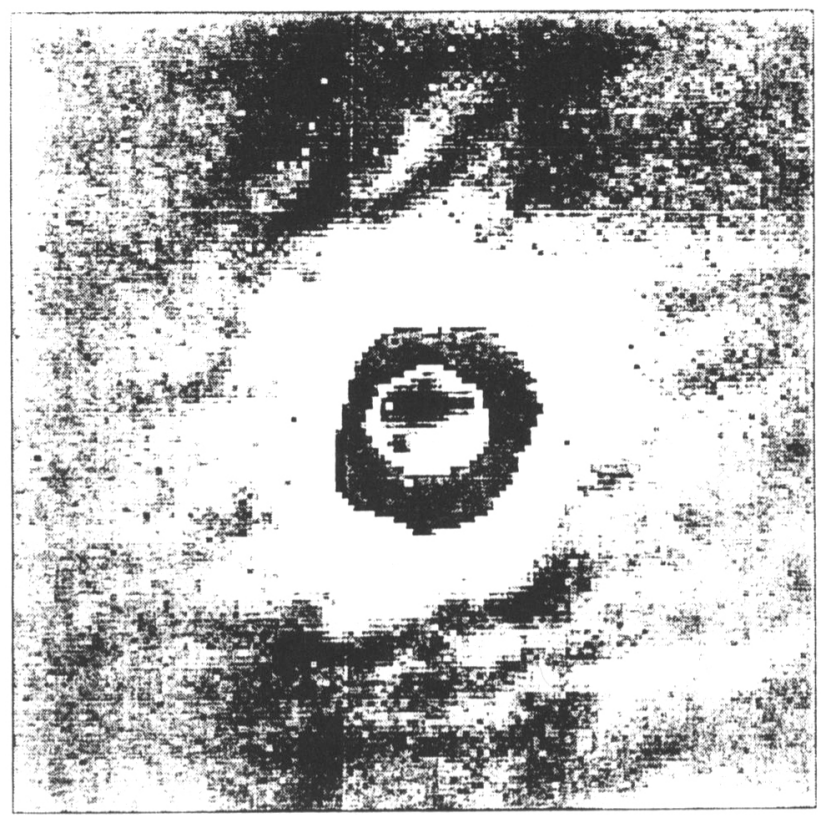

Fig. 2. The residual image obtained as the difference of two consequtive H-band observations. The change in the scattered light component is visible as a wavy pattern in this picture. Its amplitude is about $0.2 \%$ of the change in $\mathrm{H}$ brightness near the limb of the moon during this period.

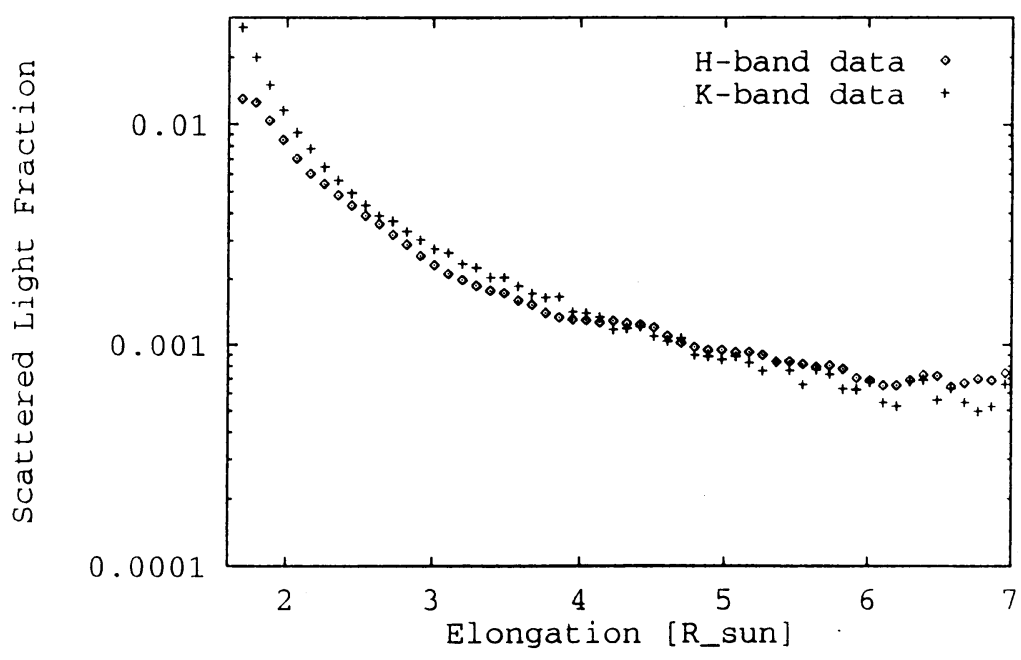

Fig. 3. This shows an estimate of the scattered light contribution to our observations. The curve is obtained from the circular average of the Fig. 2 data and has been normalized by the change in brightness near the limb. 


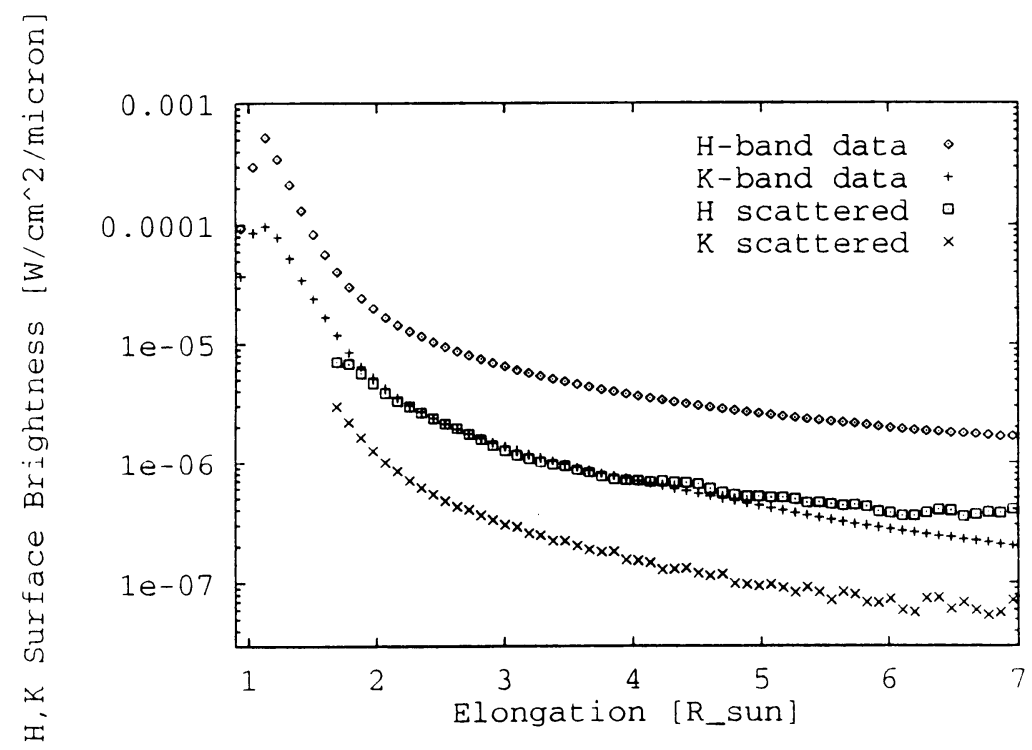

Fig. 4. The calibrated $\mathrm{H}$ and K-band surface brightness. The scattered light contribution (estimated from the data in Fig. 3) is also plotted here.

from the limb. This may explain the apparent trend to bluer colors away from the limb in Figure 4. Unfortunately the atmospheric scattered light contribution is difficult to subract from the figure 4 data - there is probably a factor of 2 normalization uncertainty in the scattered light function in addition to evidence of atmospheric variability. Nevertheless, there is no evidence of a "redder-than-solar" coronal component. Mukai (1985) also found the H and K corona to be "bluer" than solar color in a balloon observation.

We have combined the centered short and long exposure K-band data with the offset frames in order to construct a composite image that shows the coronal surface brightness from the limb of the moon out to about $14 R_{\odot}$. The offset images were obtained by pointing the instrument to the west by approximately $9 R_{\odot}$. The angular offset was accurately determined after the eclipse from digitized frames obtained with the camcorder. The IR frames were merged by finding the best linear regression between corresponding pixels in the overlapped region of each frame. The intensity scale and offset derived in this way were consistent with our uncertainty in the expected scale and sky background variations between frames. The scale change resulted from exposure time differences but was also accurately determined by this regression procedure. The resulting image is shown in Figure 5 using a logarithmic intensity scale. The mean K-band brightness and the $\mathrm{K}$ and $\mathrm{F}$ coronal model of Koutchmy and Lamy (1985) are plotted in Figure 6. Several points should be noted: First, neither the model nor data have been rescaled before plotting these 

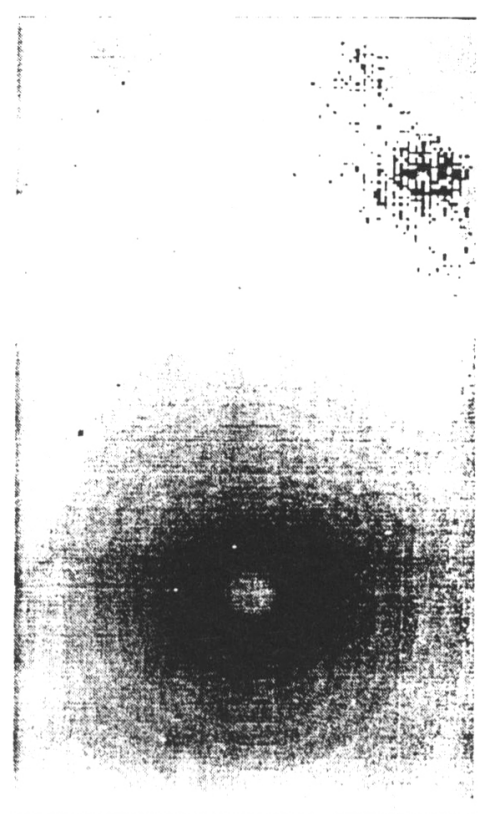

Fig. 5. The offset $\mathrm{K}$ band data has been combined with the centered data to construct this composite image which shows the corona out to $14 R_{\odot}$ in the western direction. With a rotational orientation accuracy of about 1 degree, the north direction is to the left. The greyscale of this image is logarithmic and the darkest shade is approximately $10^{4}$ brighter than the lightest shade.

curves. Second, both the data and model show a "break" in the surface brightness near $2.5 R_{\odot}$ beyond which the $\mathrm{F}$ corona dominates the $\mathrm{K}$ corona surface brightness. Lastly, there is an apparent scale difference between the model and data, in the sense that the data is everywhere brighter than the model predictions. That this is simply a scale change is demonstrated by Figure 7 which shows the data scaled by 0.5 . Note that data beyond $8 R_{\odot}$ are easily affected by small changes in the assumed background or zero level and have not been plotted here. Nevertheless, it is clear that the IR data are consistent with the visible wavelength coronal model and show no anomalous surface brightness bumps out to at least $8 R_{\odot}$.

It is clear from Figure 5 that coronal streamers can be observed in the infrared, much the same as in visible wavelengths. Figure $8 \mathrm{a}, \mathrm{b}$ shows images of the fractional deviation in $\mathrm{H}$ and $\mathrm{K}$-band light from the mean radial brightness profile. These "residual" images clearly show the aspherical structure in the corona. Since this coronal component results from "colorless" Thomson scattering of photospheric light from hot electrons, the visible structure should be independent of wavelength (as it is).

While the $\mathrm{K}$ corona should be linearly polarized, in a direction tangent to the nearest limb point, a local $\mathrm{F}$ coronal component may also be polarized. Thus, a search for anomalous polarization "rings" might also provide evidence of a local 


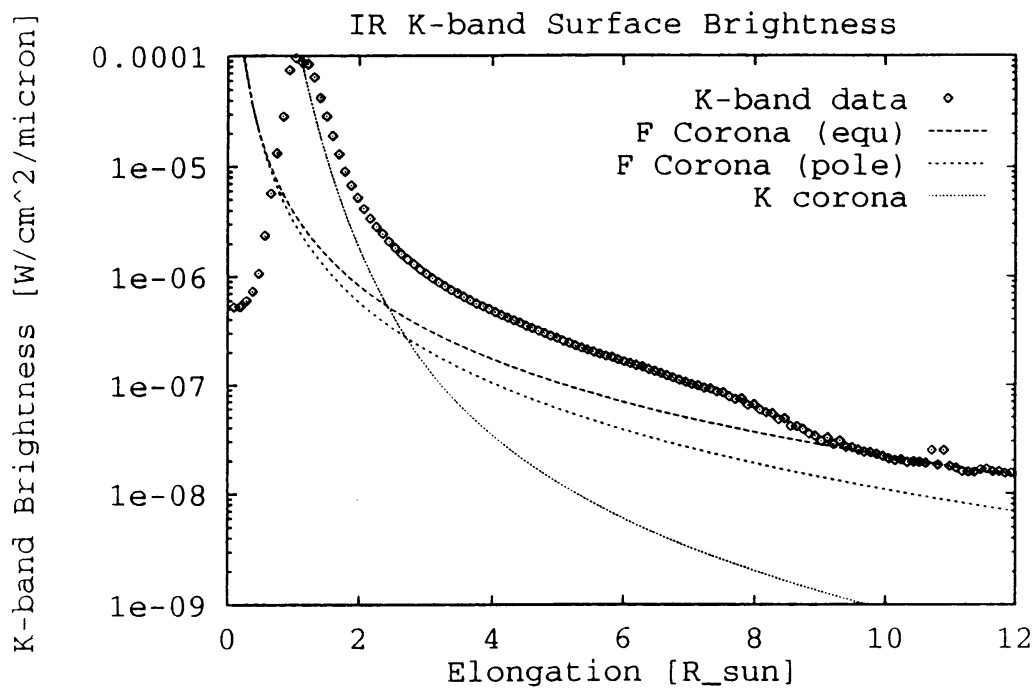

Fig. 6. The mean K-band brightness and the Koutchmy-Lamy $\mathrm{K}$ and $\mathrm{F}$ coronal model are plotted here.

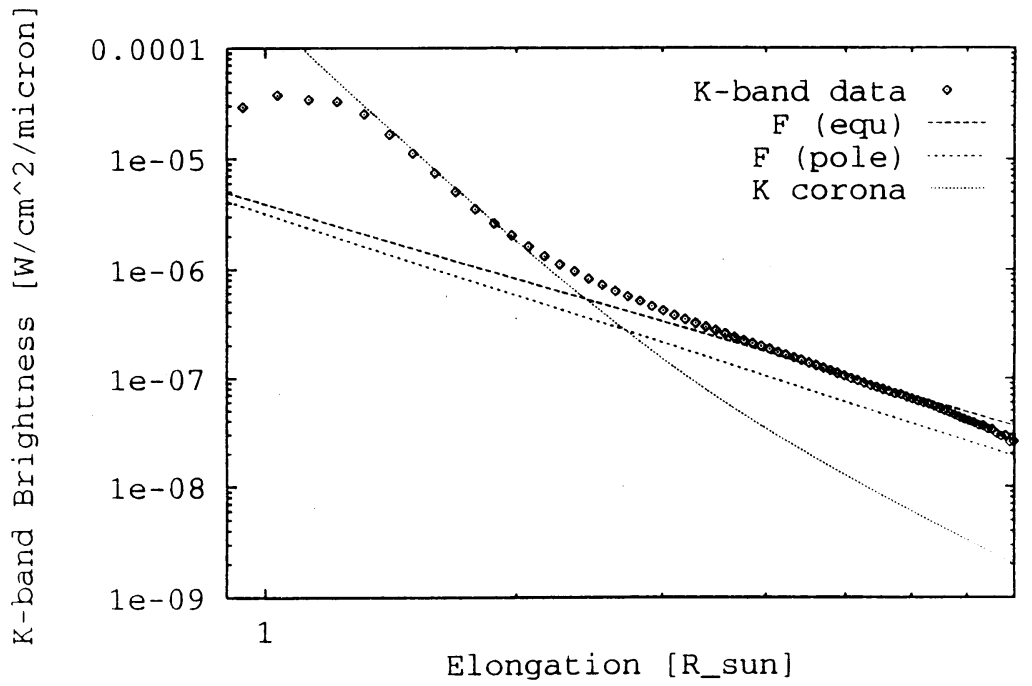

Fig. 7. This shows a comparison of the $\mathrm{K}$ data and the coronal model after allowing for a scale or calibration uncertainty in the data. 

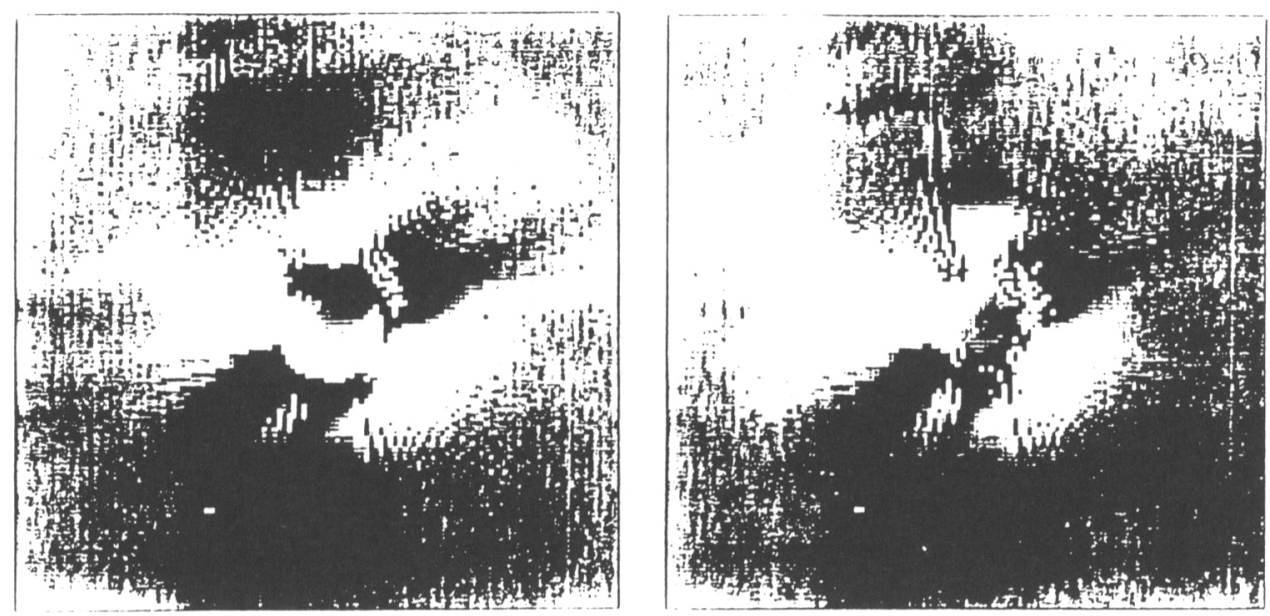

Fig. 8. These are images that show the residual structure in the H (Fig. 8a) and K-band (Fig. $8 \mathrm{~b}$ ) data. They were obtained by subtracting the circularly averaged surface brightness profile.

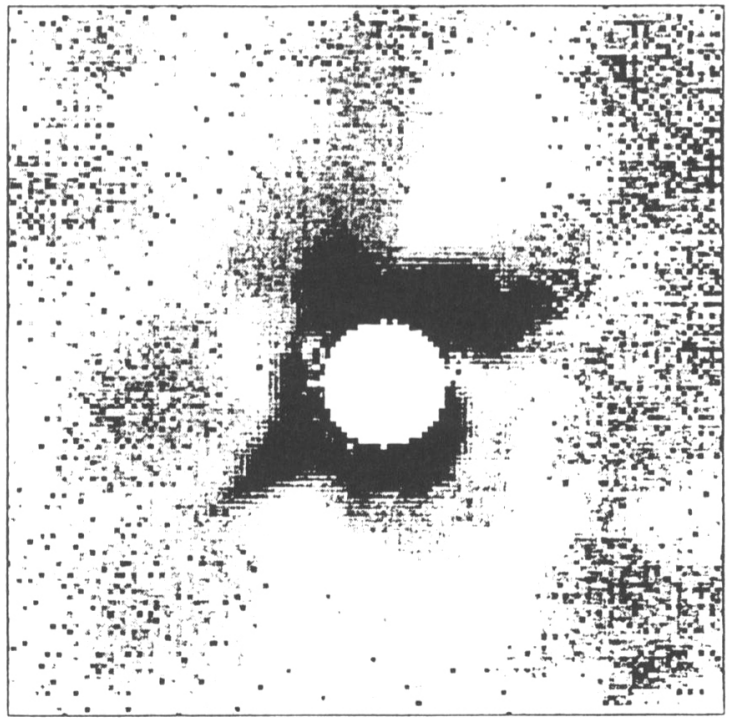

Fig. 9. This shows the mean J-band polarization fraction. The greyscale is linear in polarization where the darkest shade represents polarization of 0.4 and the lightest is a value of 0.05 . 


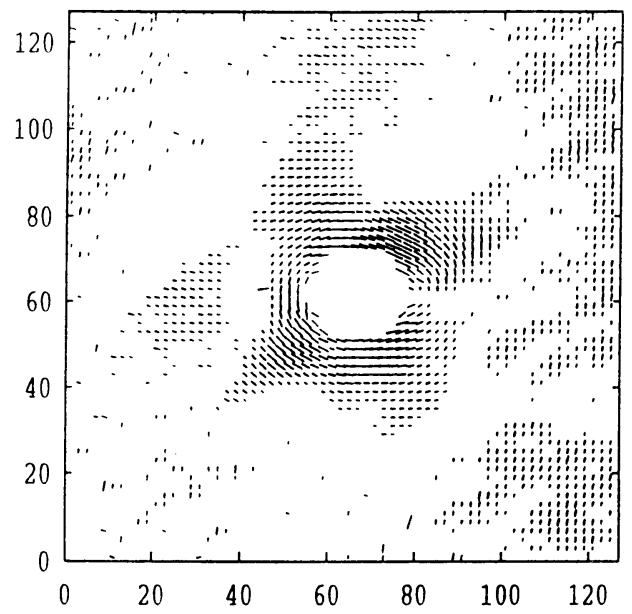

Fig. 10. The direction and magnitude of the polarization is indicated graphically in this diagram. The length of each line segment is proportional to the polarization fraction, while its direction shows the polarization plane.

F corona. Thus, J-band data were obtained through three different fixed linear polarizers, oriented at three fixed angles. Knowing these angles and the measured J-band surface brightness at each pixel we calculated the polarization fraction and direction in the image. Figure 9 is a map of the J-band polarization while Figure 10 shows the corresponding polarization direction in those pixels with polarization fraction greater than 0.1 . Figure 11 shows the mean polarization versus distance from the sun.

The rapid drop in polarization near $2 R_{\odot}$ is consistent with the break in K-band light we saw in Figure 6 which we interpreted as the "boundary" of the $\mathrm{K}$ corona. The mean polarization results are in good agreement with the data reviewed by Koutchmy and Lamy (1985), although it is clear from Figure 9 that the mean polarization is dominated by the complex aspherical shape of the streamers. The polarization direction is tangent to the limb, as we expect for Thomson scattering, and where it deviates the polarization is small and probably insignificant.

The plane of the ecliptic is inclined about 9 degrees CCW of vertical in these images. By subtracting the average radial light profile from the data we can more easily see the elliptical "zodiacal" light. Figure 12 shows such a residual image. A smooth, but flattened, coronal component is apparent beyond the $\mathrm{K}$ corona. This is not a local "F corona" since it not redder than solar color and lacks any significant polarization. Furthermore, although patchy scattered light variations limit our measurements, the apparent equatorial excess is consistent with the "zodiacal" flattening of the $\mathrm{F}$ corona described by the Koutchmy-Lamy model. According to their model the difference between the polar and equatorial K-band surface bright- 


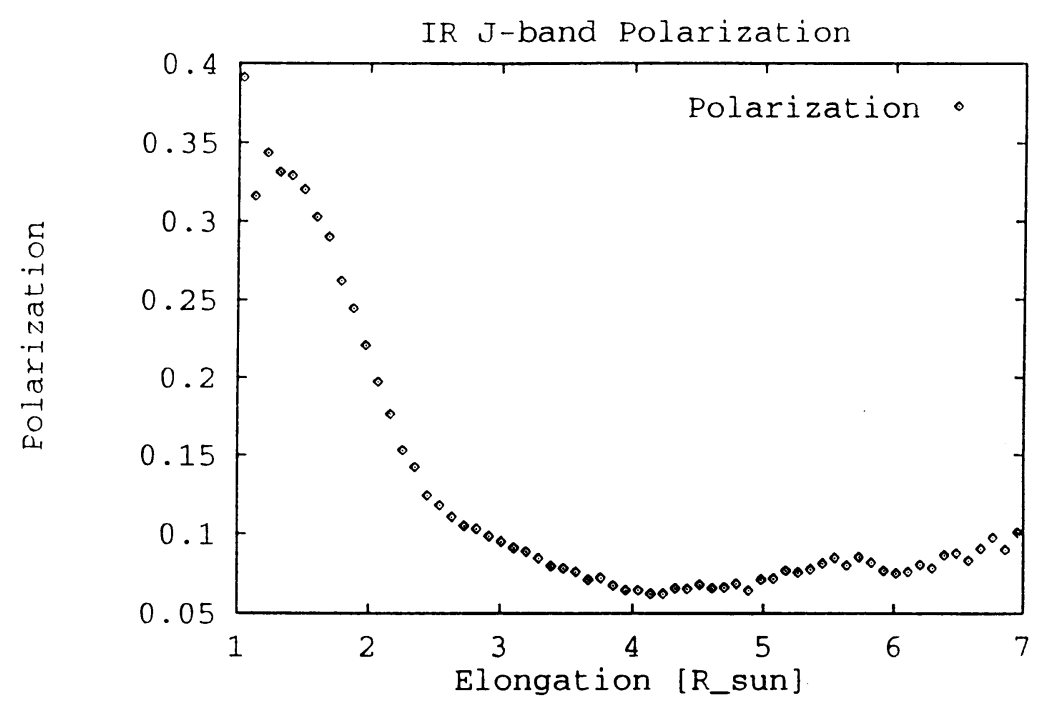

Fig. 11. The average polarization is plotted versus distance from the sun in this graph.

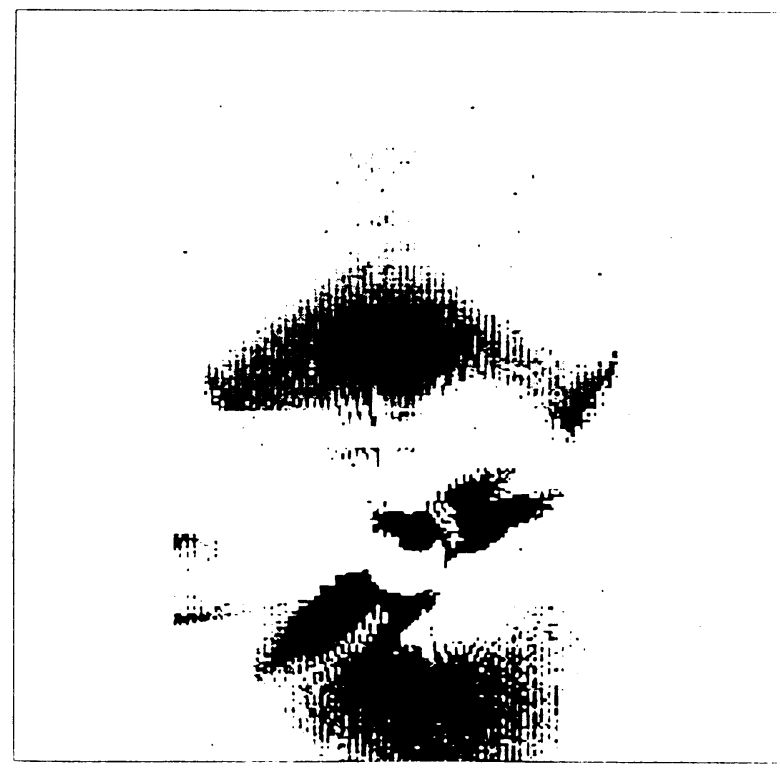

Fig. 12. A residual K-band image from the data in Figure 5 shows the ecliptical excess associated with the "zodiacal" light. This image was obtained by subtracting the circularly averaged data from Figure 5. The greyscale is linear in intensity. The range between the brightest (dark shades) and the dimmest pixel is $3.7 \times 10^{-8} \mathrm{~W} \mathrm{~cm}^{-2} \mathrm{ster}^{-1} \mu \mathrm{m}^{-1}$. 
ness near $8 R_{\odot}$ should be about $10^{-8} \mathrm{~W} \mathrm{~cm}^{-2}$ ster $^{-1} \mu \mathrm{m}^{-1}$, which is consistent with the ecliptical brightening seen in Figure 12.

\section{Conclusions}

Although sky conditions at Mauna Kea during the 1991 eclipse were not ideal, we succeeded in observing the $\mathrm{K}$ and $\mathrm{F}$ corona using a new infrared array system. Our observations through broad-band $\mathrm{J}, \mathrm{H}$ and $\mathrm{K}$ filters reveal an infrared corona with no surprises. J-band polarization data and $\mathrm{H}$ and $\mathrm{K}$ surface brightness observations clearly show the inhomogeneous structure in the $\mathrm{K}$ corona and the ecliptical flattening of the "zodiacal" light. While we detect the flattening of the non-local F corona with a surface brightness amplitude of about $10^{-8} \mathrm{~W} \mathrm{~cm}^{-2} \mathrm{ster}^{-1} \mu \mathrm{m}^{-1}$, we see no evidence of a circumsolar, local $\mathrm{F}$ corona with a brightness amplitude near $10^{-7}$ $\mathrm{W} \mathrm{cm}^{-2}$ ster $^{-1} \mu \mathrm{m}^{-1}$ (MacQueen 1968). If there is a local dust component to the corona it must now be considerably smaller than it was in the past.

\section{Acknowledgements}

This experiment was supported by the Laboratoire d'Astrophysique Spatial, the Canada-France Hawaii consortium, and Michigan State University. We are grateful to David Hoadley and Nathan Blair for help with the camera software and polarization analysis. The IR camera system was developed in collaboration with the University of Wyoming and Haverford College through the Astrophysical Research Consortium.

\section{References}

Koutchmy, S. and Lamy, P.: 1985, in R.H. Giese and P. Lamy (eds.), Properties and Interactions of Interplanetary Dust, p. 63.

McPherson, M., Lin, H. and Kuhn, J.R.: 1992, Solar Phys. 139, 255.

MacQueen, R.M.: 1968, Astrophys. J. 154, 1059.

Mampaso, A., Sanchez-Magro, C., and Buitrago, J.: 1982, in W. Fricke and G. Tedeki (eds.), Sun and Planetary Systems, p. 257.

Mukai, T.: 1985, in R.H. Giese and P. Lamy (eds.), Properties and Interactions of Interplanetary Dust, p. 59.

Peterson, A.W.: 1963, Astrophys. J. 138, 1218.

Wyatt, S., Whipple, F.L.: 1950, Astrophys. J. 111, 134. 